\title{
Enseignement de l'architecture et marche du travail
}

\author{
RICARDO VERGĖS-ESCUIN*
}

\section{RESUME}

La présent recherche comprend trois niveaux. D'abord, mise au point d'un instrument mesurant l'offre et la demande d'architectes. Ensuite, application au cas précis du Québec, de manière à mettre en évidence les problèmes d'insertion économique de la profession. Enfin, prospective des variables explicatives de la demande en vue de leur intégration dans un système de planification de l'enseignement. En particulier, les prévisions d'investissement immobilier sont dérivées de l'évolution probable du stock immobilier selon la population, le revenu et la préférence de consommation. Ce dernier développement confère à l'étude un intérêt pour toutes disciplines et activités économiques concernées par la construction immobilière et le cadre bâti.

\begin{abstract}
This study has been carried out on three levels. Firstly, the precise definition of an instrument for measurement of the supply and demand of architects. Following this, the application of the measurement to the specific case of Quebec in such a way as to clearly pose the questions concerning the economic integration of the profession. Finally, a forecast of the variables that explain the demand with a view to their integration into a system of educational planning. In particular, forecasting of real estate investment are derived from the probable evolution of available real estate broken down according to population, revenue and consumer preference. This last section makes the study of interest to those in all economic disciplines and activities concerned with real estate construction and building sites.
\end{abstract}

\section{INTRODUCTION}

C'est un fait que depuis quelques années il y a pléthore dans la majorité des disciplines.

Le marché du travail ne parvient pas à absorber la masse de diplômés produite par

l'explosion scolaire ${ }^{(1)}$. Il s'ensuit le phénomène bien connu du chômage instruit (2) 
accompagné bien souvent de puissants flux d'émigration. . .ou de retour aux études.

Parmi ces disciplines, l'architecture est, bien sûr, aux prises avec de tels problèmes. Mais dans la plupart des pays, sa situation est aggravée par un afflux grandissant de jeunes persuadés d'y trouver un champ d'action idoine pour améliorer l'environnement.

La réalité est que les classes jeunes d'architectes se trouvent dans l'incapacité de s'intégrer dans la profession et par conséquent, de réaliser leurs aspirations. Ceci engendre toutes sortes de frustrations sans compter le gaspillage que représente sur le plan social, la mise à l'écart d'aussi vastes ressources humaines (3).

Est-ce dire que ces ressources sont, de fait, superflues? Nous ne le croyons pas, vu les insuffisances partout décriées des résultats de l'actuel système de production du cadre bâti ${ }^{(4)}$. Nous sommes plutôt persuadés qu'il faut trouver une bonne partie des causes de cet état de fait dans les déficiences de l'actuel système d'enseignement de l'architecture. Celui-ci, en effet, n'apparaît pas en mesure de répondre de manière adéquate à certains critères de nature économique, seuls susceptibles de sortir cette profession de son état de crise ${ }^{(5)}$.

Le présent article a donc comme premier but d'analyser le rapport enseignementmarché du travail en architecture. Le deuxième but est de formuler les bases d'un élargissement professionnel socialement utile et économiquement viable.

\section{LES ARCHITECTES DANS L'ACTIVITE ECONOMIQUE}

Une certaine ambiguîté règne à propos de la définition de l'architecte. En fait, nous avons été conduits à adopter la seule définition économiquement praticable, à savoir celle de la classification des professions ${ }^{(6)}$. Nous avons dû préférer cette définition à celles de nature corporative ou éducationnelle destinées essentiellement à délimiter le mode libéral d'exercice de la profession.

La masse d'architectes actifs "peut être assimilée à un réservoir dont le niveau d'équilibre s'élève au fur et à mesure qu'augmentent les besoins de l'économie, mais dont le niveau effectif se situe généralement en dessous (situation déficitaire) ou en dessus (situation excédentaire)"(7).

Il ressort de cette analogie, non seulement le mécanisme de l'offre et de la demande que nous cherchons à mesurer mais encore l'ensemble des facteurs en jeu.

Le premier de ces facteurs est évidemment le contenu du réservoir, c'est-à-dire l'ensemble des architectes actifs au moment de la mesure initiale. Deuxièmement, il y a le débit d'alimentation, soit les nouveaux architectes en provenance des institutions pédagogiques autochtones ou d'autres pays. Or, l'effectif initial subit une déperdition naturelle en raison de la retraite et de la mortalité: c'est donc le troisième facteur. Enfin, le quatrième est constitué par le relèvement plus ou moins continu de la "digue" du réservoir à savoir la variation de la demande d'architectes.

Un certain nombre de situations typiques résultent du jeu de ces quatre facteurs. Si le recrutement se tarit, la profession disparaîtra (certaines communautés religieuses. ..). Si le débit compense les pertes de mortalité et retraite, la profession stagnera (députés élus d'une législature. . .). Si le débit est tel que le niveau demeure en dessous de la demande, il y aura déficit (certaines professions manuelles dévalorisées par rapport aux emplois tertiaires. . .). Si le débit est réglé sur la demande prévue, il y aura équilibre (professions 


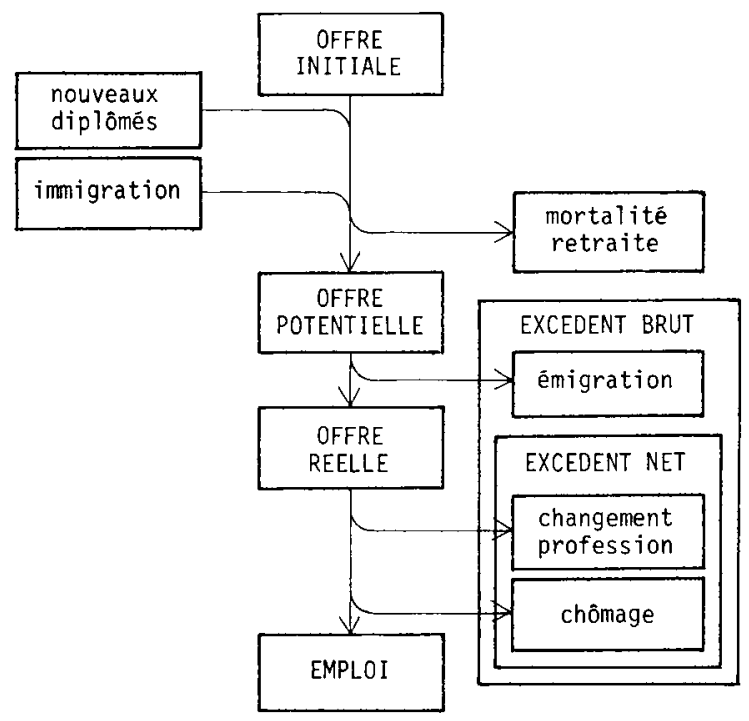

Graphique 1. Concepts d'offre et de demande d'architectes

contingentées. . .). Enfin, si le débit est trop élevé, il y aura excédent, ce dernier pouvant être décroissant, stationnaire ou croissant.

C'est sans aucun doute cette dernière situation qui prévaut dans bien des pays en matière d'architecture puisque le débit d'alimentation (éducation, immigration) est trop puissant par rapport à la demande. Le surplus se déverse donc vers des réservoirs d'attente (chômage), vers d'autres secteurs de l'économie (changement de profession) ou vers d'autres pays (émigration).

\section{Méthodologie de la mesure de l'offre}

Nous avons mis au point une méthodologie d'analyse à l'occasion de l'étude du cas du Québec. Pour mesurer l'offre potentielle d'architectes, nous avons utilisé un modèle récursif de type classique ${ }^{(8)}: \mathrm{E}=(1-\lambda) \mathrm{E}_{\mathrm{t}-1}+\mathrm{n}_{\mathrm{t}}$.

Dans ce modèle $\mathrm{E}_{\mathrm{t}}$ représente l'effectif recherché, $\lambda$ le taux de départ par mortalité et retraite et $\mathrm{n}$ le débit des maisons d'enseignement et des canaux d'immigration. Ce modèle a été appliqué séparément à chacun des flux constitutifs de l'offre, soit l'offre potentielle, les nouveaux diplômés et l'immigration.

Les données sur l'offre initiale ont été fournies par les recensements. Celles sur les nouveaux diplômés et les effectifs universitaires ont été compilées par les maisons d'enseignement. Enfin, les données sur l'immigration ont été fournies par les services administratifs correspondants. Dans chaque cas, le calcul démographique appliquant $\lambda$ a été effectué à l'aide de diagrammes de Lexis ${ }^{(9)}$ par application des taux de retraite observés par les recensements et des taux de mortalité de la population masculine du Québec.

De toute notre étude se dégage le fait que l'offre potentielle d'architectes fonctionne comme un système autonome alimenté par des sources parfaitement mesurables mais 


\begin{tabular}{|l|c|c|c|c|}
\hline \multicolumn{1}{|c|}{ secteur } & $\begin{array}{c}\text { constante } \\
\mathrm{a}\end{array}$ & $\begin{array}{c}\text { pente } \\
\mathrm{b}\end{array}$ & $\begin{array}{c}\text { Student } \\
\mathrm{T}\end{array}$ & $\begin{array}{c}\text { corrélation } \\
\mathrm{r}\end{array}$ \\
\hline rēsidentiel collec. & 1.35678 & .69010 & .82423 & .93609 \\
commercial & -4.99299 & 1.06897 & -.72559 & .63695 \\
industriel & .17369 & .62498 & .14345 & .85101 \\
éducation & 5.78336 & .87594 & 2.52860 & .86685 \\
Santé & -2.95828 & 2.07733 & -.87383 & .67329 \\
réligion & 1.50934 & .69592 & 3.00725 & .79042 \\
édifices publics & 5.29842 & 1.76854 & .20078 & .71550 \\
\hline
\end{tabular}

Graphique 2. Caractéristiques du modèle PACC

difficilement prévisibles. En effet, en l'absence de structures appropriées de contingentement, il est impossible de prévoir quelle sera la demande future d'inscription dans les institutions d'enseignement et encore moins quel sera le flux d'entrée de diplômés étrangers.

\section{Méthodologie de la mesure de la demande}

Il est rare de disposer de données annuelles sur l'ensemble de l'emploi. Toutefois, les recensements nous renseignent sur le nombre d'architectes actifs travaillant, soit dans les bureaux professionnels privés, soit ailleurs. Comme ces derniers représentent une faible proportion du total ( 15 à $30 \%$ selon les pays ${ }^{(10)}$ ), et que les données correspondantes ne sont disponibles que tous les dix ans, il est important de mettre l'accent sur l'étude de la demande annuelle en provenance des bureaux d'architecte.

Tel est le cas aux Etats-Unis et au Canada. Comme par ailleurs on n'a des données fiscales sur les architectes que pour un nombre excessivement restreint d'années, nous avons construit un instrument économétrique, le modèle PACC, pour étudier cette demande. Le modèle consiste en une batterie d'équations linéaires simultanées ${ }^{(11)}$ mesurant la variation structurelle des projets d'architecture effectués par les bureaux d'architecte en fonction de la variation également structurelle des travaux immobiliers selon les secteurs ${ }^{(12)}$. Le tableau ci-après fournit les résultats obtenus (13).

On peut remarquer, partant des résultats du modèle ci-dessus que le jeu combiné des constantes et des pentes fait que les droites de régression des quatre derniers secteurs se situent "en amont" des trois secteurs précédents: les secteurs institutionnels sont ceux dont les édifices requièrent le plus les services d'un architecte. Par ailleurs, les tests de Student sur les constantes des équations montrent que dans l'ensemble, celles-ci ne sont pas significativement différentes de zéro: si par exemple, un secteur immobilier se développe relativement aux autres secteurs, on constate une variation pratiquement proportionnelle dans les volumes relatifs des projets de ce type chez les architectes. Enfin, on peut considérer les coefficients de corrélation comme satisfaisants surtout si l'on tient compte du fait que les deux séries de données ont été collectées par des organismes absolument distincts et avec des procédures très éloignées l'une de l'autre quant aux moyens mis en oeuvre.

Les séries structurelles de la construction immobilière ont été soumises au modèle PACC ce qui a fourni, aux variables aléatoires près, la structure procentuelle des projets d'architecture dans les bureaux d'architecte. Or, en assumant l'hypothèse que dans aucun secteur le montant monétaire des projets d'architecture ne peut dépasser celui des travaux 
de construction, il est possible de déduire, par analyse algébrique, la valeur maximale de l'ensemble des projets effectués par les bureaux. En effet, le rapport entre cette valeur maximale et celle de l'ensemble des travaux de construction est égale à l'inverse du plus petit rapport entre les pourcentages des secteurs homologues d'une part de la construction (déduits des données disponibles) et d'autre part des projets d'architecture (calculés à l'aide du modèle PACC).

Les estimations ainsi obtenues peuvent être confrontées avec les données, même fragmentaires, de la fiscalité. Dans le cas des Etats-Unis et du Québec (11), nous avons pu vérifier que les revenus professionnels bruts déclarés par les propriétaires des bureaux (patrons et associés) représentaient sensiblement les honoraires correspondant aux montants des projets estimés selon PACC. Enfin, pour transformer les estimations de la valeur des projets en termes d'emploi, on doit utiliser des hypothèses de productivité basées sur les niveaux d'emploi et de revenu des architectes aux années de recensement, niveaux qui peuvent ensuite être interpolés annuellement.

\section{Méthodologie de l'ajustement de l'offre à la demande}

Les écarts entre l'offre potentielle et la demande d'architectes donnent lieu à des ajustements positifs (excédent) ou négatifs (déficit). On a déjà pris pour acquis que c'est la situation excédentaire qui reflète de plus en plus le contexte actuel de la profession dans la plupart des pays. C'est pourquoi on peut se limiter à l'analyse de l'excédent positif.

En pratique, l'écrat excédentaire entre l'offre et la demande d'architectes se résout par le chômage, le changement de profession (y compris le retour aux études) et l'émigration, chacun de ces flux devant être mesuré à l'aide d'un modèle récursif comme celui de l'offre ci-avant.

\section{Une étude pilote: le cas du Québec (11)}

La méthodologie qui vient d'être décrite a été appliquée d'une manière globale au cas du Québec. Ceci a posé deux problèmes particuliers. Le premier concernait la transposition générale du modèle PACC à d'autres pays que les Etats-Unis. Dans le cas du Québec, nous avons effectué plusieurs tests statistiques de similarité sur la structure de la construction immobilière et sur la structure de la profession d'architecte dans les deux pays. Le deuxième problème était la mesure des flux migratoires interprovinciaux. L'exode vers les autres provinces a pu être mesuré grâce aux enquêtes sur le déplacement de population active effectuées dans le cadre des recensements décennaux. Quant à l'émigration, elle a pu être mesurée en pondérant les chiffres concernant l'entrée d'architectes aux Etats-Unis en provenance du Canada, selon une méthode mise au point récemment par Statistique Canada $(14)$.

Ce graphique appelle plusieurs commentaires. Tout d'abord l'extraordinaire expansion de l'offre depuis le milieu des années 60. Qu'elle ait été occasionnée par l'offre interne (nouveaux diplômés) ou externe (immigration), la cause en est l'afflux croissant de jeunes vers les études d'architecture au Québec comme partout ailleurs ${ }^{(15)}$.

Par ailleurs, si on examine l'allure de la demande dans le graphique 3, on constatera deux phénomènes. D'une part l'existence de fortes fluctuations cycliques ${ }^{(16)}$, d'autre part, un certain fléchissement de la tendance à long terme de la demande d'architectes. 


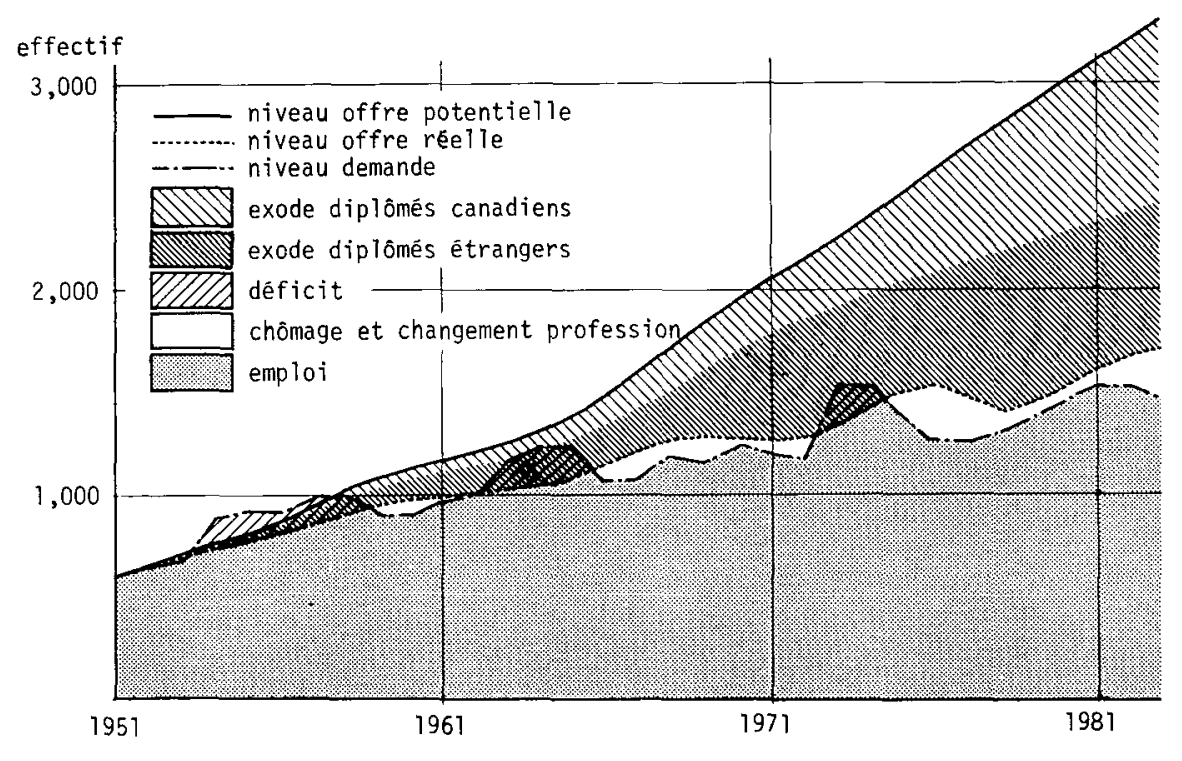

Graphique 3. Offre et demande d'architectes au Québec

Il est clair que ce fléchissement est parallèle à celui de la construction immobilière. Nous reviendrons ci-après sur ce phénomène d'importance capitale mais d'ores et déjà, on peut observer que par delà les hauts et les bas cycliques, la demande d'architectes tend à se stabiliser à un niveau proche du niveau actuel.

Enfin, l'écart considérable entre offre potentielle et demande pose la question de savoir où est passée la différence. Notre étude montre que dans le cas spécifique du Québec, la plus grande partie de l'excédent brut s'est transformée en flux migratoire sortant à destination des Etats-Unis ou des autres provinces canadiennes, l'Ontario en particulier. La prépondérance des variables migratoires dans l'excédent met en relief la faible élasticité de substitution des architectes. En effet, chaque crise d'emploi, en particulier immédiatement après chaque "haut" de la construction, s'est soldée par un exode d'effectifs quasi égal à la totalité de l'excédent en cours de formation. Il n'est donc pas étonnant qu'il puisse y avoir - paradoxalement - déficit d'architectes lors des "reprises" de la construction du genre de celles qui ont eu lieu au Québec au milieu de chaque décennie.

Même circonscrite au cas du Québec, une telle situation est illustrative des distorsions qui existent entre les aspects au moins quantitatifs de la formation des architectes et leur insertion dans l'activité économique. De plus en plus, étudiants, corporations professionnelles, pouvoirs publics de maints pays aussi bien que les organisations internationales concernées s'inquiètent de ce problème aux conséquences sociales considérables ${ }^{(17)}$.

\section{LES VARIABLES EXPLICATIVES DE LA DEMANDE D'ARCHITECTES}

Le modèle PACC montre que la demande d'architectes est une fonction de l'investissement immobilier. Mais, existe-t-il des prévisions solides en matière de construction immobilière? 


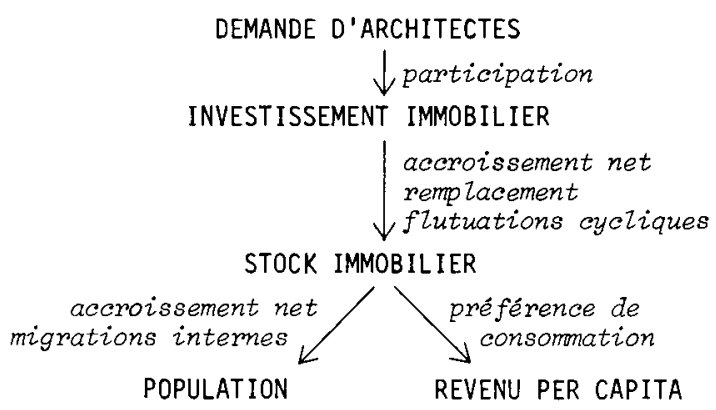

Graphique 4. Variables explicatives de la demande d'architectes.

La réponse apparaît plutôt négative. Cela tient à la nature même des courants qui ont alimenté la littérature économique sur le sujet et qui ont porté l'attention soit sur les tendances et cycles séculaires, soit sur les prévisions à très court terme ${ }^{(18)}$.

Or, on dispose actuellement de séries sur le stock de capital immobilier, ce qui offre une base idoine pour l'élaboration de prévisions à moyen et long terme. En effet, le rôle de l'investissement immobilier est d'accroître et de remplacer le stock immobilier pour l'usage d'une population donnée et ce, en fonction de son niveau de revenu et de ses préférences de consommation. Il doit donc être possible de dériver les tendances de l'investissement immobilier à partir de celles constatées dans la consommation du stock.

Dans le schéma ci-dessous, le système recherché a été représenté par une chaîne de variables (majuscules) dépendantes les unes des autres (sens des flèches) selon des fonctions ou sous-modèles spécifiques (italiques).

\section{Population et stock immobilier}

Si l'on fait abstraction des inégalités bien connues en matière d'usage immobilier (19), il y a lieu de considérer qu'uń accroissement démographique entraîne un accroissement proportionnel de stock immobilier. Ainsi, un apport absolu de nouvelle population sur le plan national entraînera sûrement la construction de nouveaux logements, hôpitaux, commerces, etc. D'autre part, des migrations comme celles qui tendent vers les banlieues lointaines au détriment des centres villes, occasionnent des accroissements relatifs de population qui se répercutent à leur tour sur le volume du stock immobilier.

En d'autres termes, la population est la variable explicative première du stock immobilier. En abrégeant sur les détails, on peut donc initier la modelisation de la demande d'architectes en réduisant les différentes variables à leur valeur per capita.

\section{Niveau de revenu et stock immobilier}

Que se passe-t-il lorsqu'une population donnée voit son revenu augmenter? Il est évident qu'elle aura tendance à accroître le niveau de son stock immobilier. Cependant, nos recherches actuelles ${ }^{(20)}$ sur l'évolution historique des stocks bruts selon les secteurs immobiliers au Canada et aux Etats-Unis ${ }^{(21)}$ montrent que la part du revenu qui est affectée au cadre bâti ne revêt pas un caractère de proportionnalité statique. Ainsi, au seuil de la phase de croissance industrielle, l'investissement dans le stock immobilier est proportionnellement plus élevé que lorsque la société s'approche de la phase "post- 


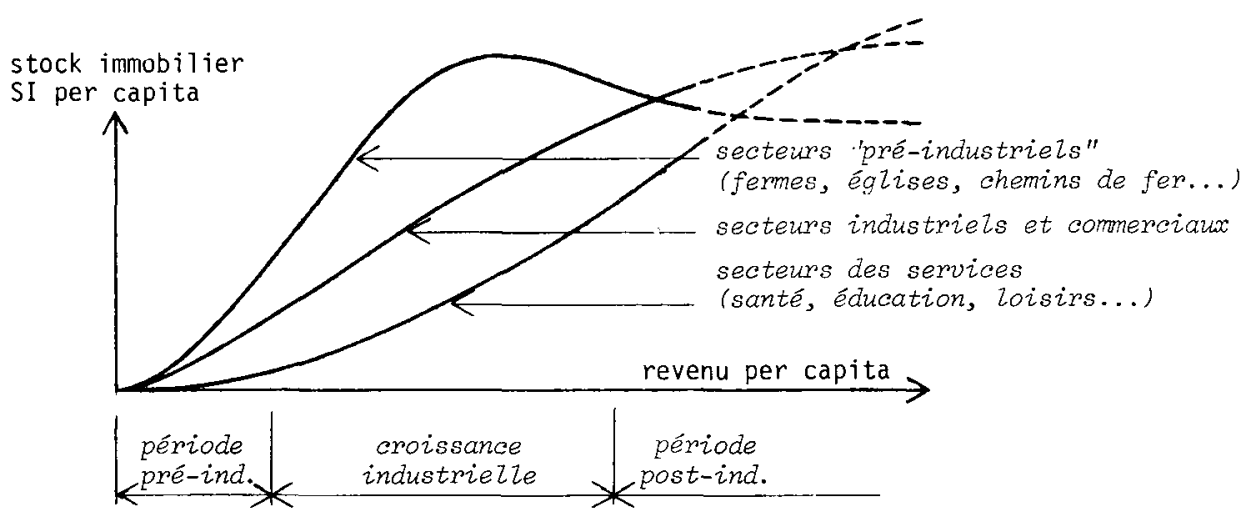

Graphique 5. Evolution des secteurs du stock immobilier per capita par rapport au revenu per capita.

industrielle" out de "maturité". En d'autres termes, l'élasticité du stock par habitant par rapport au revenu également par habitant, croît au début de la croissance économique et décroît à l'approche de la phase post-industrielle.

Tout se passe comme si à partir d'un certain niveau de développement - c'est-à-dire d'industrialisation et d'urbanisation - une société s'estimait satisfaite du niveau atteint par le stock immobilier et préférait affecter ses ressources dans d'autres secteurs d'investissement ou de consommation. La mise en évidence de ce niveau de saturation suggère que la constitution du stock immobilier obéit à des fonctions "logistiques" dépendantes des phases de la croissance économique et des secteurs immobiliers considérés.

Nous ne discuterons pas ici du problème de la réversibilité de ces fonctions. Car, comme l'ont montré Kuznets, Rostow et bien d'autres, si le revenu entraîne l'épargne et celle-ci la capitalisation, l'inverse est aussi vrai. Cependant, ce que nous recherchons à travers ces fonctions n'est pas la causalité mais la concomitance.

\section{Stock et investissement immobilier}

Il est évident que l'accroissement du stock immobilier existant est obtenu grâce à la construction immobilière. Il est non moins évident aussi que, dans la plupart des pays industrialisés, l'expansion démographique tend à ralentir et parfois même à stagner (22). Dès lors, comment évoluera cet investissement dans le cas d'une population numériquement stable? En fait, la partie qui sert à l'accroissement net du stock évolue comme la dérivée de la fonction du stock (graphique 5). Par conséquent, cette partie de l'investissement aura tendance à diminuer à l'approche de la phase post-industrielle après avoir atteint son sommet en pleine croissance économique. Le graphique suivant montre cette évolution.

Toutefois, le stock ainsi constitué n'est pas éternel. Il doit être remplacé au terme de $\mathrm{L}$ années moyennes de vie utile ${ }^{(23)}$. Et la partie de l'investissement immobilier qui assure ce remplacement est évidemment égale à l'investissement (en monnaie constante) de l'année $\mathrm{t}-\mathrm{L}$.

Mais l'ensemble de l'investissement immobilier ne se réalise année après année de manière uniforme. Il connaît au contraire des cycles dont la fréquence et l'amplitude 


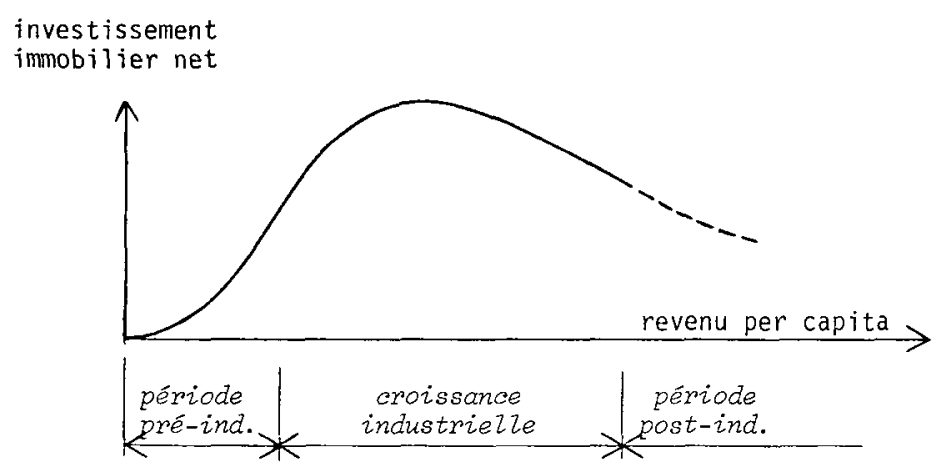

Graphique 6. Evolution de l'investissement immobilier net

peut être déterminée, par exemple, par l'analyse spectrale. Ce sont des cycles "Kuznets" (15-25 ans), "longs" (6-11 ans) et "courts" (2-4 ans) sans compter les variations saisonnières (18).

De tout cela découle que la simulation IIT niveau annuel de l'investissement immobilier résulte de l'agrégation de l'accroissement net du stock (dérivée de SI), du remplacement des bâtiments au terme de leur vie utile $\left(\mathrm{II}_{t-L}\right)$, des fluctuations cycliques $\lambda$ et du terme aléatoire $\epsilon$.

$$
\mathrm{IIT}_{\mathrm{t}}=\mathrm{f}^{\prime}(\mathrm{SI})+\mathrm{II}_{\mathrm{t}-\mathrm{L}}+\lambda(\mathrm{t})+\epsilon
$$

En définitive, si l'on retient toujours la double hypothèse d'une population stabilisée et d'un revenu par habitant croissant exponentiellement, on obtient l'expression graphique ci-dessus après transposition chronologique de la fonction IIT. Dans une perspective de stabilisation démographique, la construction immobilière neuve tend vers un niveau moyen d'équilibre.

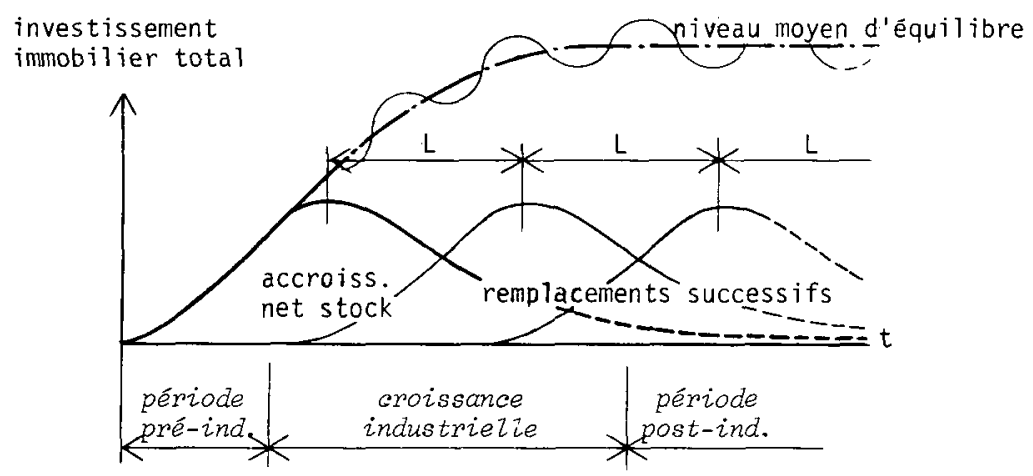

Graphique 7. Evolution de l'investissement immobilier total. 


\section{POLITIQUES ALTERNATIVES POUR LA PROFESSION D'ARCHITECTE}

\section{Planification universitaire vs "laissez-faire"}

Dans la première partie du présent texte, nous avons, à travers l'offre et la demande d'architectes, mesuré l'insertion de cette profession dans l'activité économique. De fait, il y avait une hypothèse implicite, à savoir qu'offre et demande représentent des variables totalement dissociées.

Par le passé, la confrontation sur le marché de l'offre et de la demande s'est soldée par un ajustement de signe alternativement positif et négatif. Si on rappelle que l'offre résulte de l'agrégation de l'offre initiale (OI), des nouveaux diplômés (DIP), et de l'immigration (IMM) et que la demande est une fonction de la construction neuve ou investissement immobilier (II), on peut écrire

$$
\mathrm{OI}+\mathrm{DIP}+\mathrm{IMM}=\mathrm{f}(\mathrm{II}) \pm \mathrm{AJUST} \text {. }
$$

Or, on a vu que ces derniers temps, l'ajustement est toujours positif et de plus en plus élevé, ce qui ne va pas sans poser d'énormes problèmes. Dès lors, toute planification visera nécessairement à annuler AJUST ou, en d'autres termes, à faire tendre le premier terme de l'équation vers f(II). De plus, comme en termes de contrôle, OI s'apparente plus à une constante qu'à une variable proprement dite, on peut écrire le postulat fondamental de tout système de planification professionnelle:

$$
\text { DIP + IMM f(II) }
$$

Dans un système de planification, les variables de l'offre deviennent dépendantes de la demande. Ce énoncé pose un problème d'ordre politico-social. En effet, sur quel principe peut-il être basé?

Nous savons quelle réponse l'économie classique de l'éducation a donné à cette question à partir de la nécessité sociale de rentabiliser les investissements ${ }^{(24)}$. Or l'éducation en est un. Celle-ci implique en effet des coûts directs - de plus en plus élevés d'ailleurs - plus des coûts indirects analysés comme un "manque à gagner" pendant la durée où l'étudiant cesse de contribuer à la production(3). Le "taux de rendement" (25) devient positif lorsque les revenus directs et indirects supplémentaires procurés par l'accroissement du niveau d'éducation dépassent l'ensemble des coûts de celle-ci. La démonstration faite par Edward Denison ${ }^{(26)}$ que l'éducation et la recherche de la connaissance ont été de loin les principaux facteurs de la formidable croissance économique des Etats-Unis, a conféré à cette thèse un caractère dogmatique.

Toutefois, le gonflement actuel des effectifs universitaires et l'apparition du chômage instruit viennent jeter un doute sur le caractère exclusif du rôle éconor nique de l'éducation. Ainsi, d'autres options apparaissent dont celle du rôle de consommation culturelle ${ }^{(27)}$ qui tend à s'étendre au-delà de la simple éducation "générale" comme le suggère Milton Friedman, pour englober aussi une bonne partie de l'éducation technique.

Il est clair que l'issue d'un tel débat dépendra de la société toute entière. . .sinon du contribuable! Il est probable que la planification universitaire du futur intègre les deux caractères: on ne voit pas aujourd'hui la possibilité d'une université strictement contingentée en fonction des besoins économiques ni, au contraire, d'un système d'enseignement supérieur qui tournerait "en roue libre" et ne tiendrait aucun compte desdits besoins 
comme c'est le cas en architecture. Notre but est de concevoir un mécanisme fournissant à l'enseignement - et aussi à l'immigration - les informations nécessaires à la prise de décision, laquelle, en tout état de cause, doit demeurer politique.

\section{Contingentement vs diversification}

L'optique du contingentement repose sur l'hypothèse que la fonction reliant la demande à ses propres variables explicatives (en l'occurence, l'investissement immobilier) est fixe. Or, ne serait-il pas possible de changer cette fonction? Toute la tendance appelée diversification cherche à répondre à cette question par l'affirmative ${ }^{(28)}$.

Cependant, la tentative de diversification n'a obtenu, jusqu'ici, que des résultats mitigés. Ceci mérite d'être discuté en particulier à travers le cas des Etats-Unis où la tendance s'est exprimée le plus précocement ${ }^{(29)}$.

L'indicateur de la diversification est évidement la variation de la masse d'architectes travaillant ailleurs que dans les bureaux professionnels. Pour cela, nous pouvons utiliser les tables des recensements concernant les professions selon les industries ${ }^{(30)}$. Ces tables fournissent le nombre d'architectes qui travaillent dans les industries manufacturières, dans l'industrie de la construction, dans l'administration publique, dans le secteur immobilier, dans les différentes institutions, etc., aussi bien que dans les bureaux professionels.

Toutefois, les classifications américaines des recensements montrent que le term "architects" inclut les "landscape architects" et les "naval architects" excepté en 1960. Ceci nous oblige à retrancher un certain effectif de la population d'architectes figurant respectivement aux recensements de 1940, 1950 et de 1970.

Une fois ces opérations effectuées, on se trouve disposer de données homogènes. Toutefois, la variation du nombre d'architectes travaillant dans les différentes industries doit ètre évalué rélativement à la variation de la variable explicative de l'activité architecturale. $\mathrm{Ou}$, le modèle PACC nous a montré que cette variable est essentiellement constituée par l'investissement immobilier non agricole et non "unifamilial".

PACC montre aussi que le nombre d'architectes actifs une année est fonction des investissements en valeur réelle de l'année suivante en raison de l'anticipation de l'étude par rapport à la passation du contrat de construction.

Finalement, nous avons représenté dans le graphique 8 , le nombre d'architectes travaillant dans diverses industries par rapport aux investissements immobiliers selectionnés selon les critères ci-dessus et exprimés en $\$ 1958^{(32)}$.

On peut remarquer en 1970 une faible reprise de la diversification. En effet, si les emplois d'architecte selon des industries avaient crû entre 1960 et 1970 proportionnellement à la construction immobilière en $\$$ constants, on aurait observé en 1970 quelques 35,700 architectes des bureaux. En fait, on en a observé 34,500 et 12,500 respectivement. Donc, la diversification aurait procuré environ 4,000 emplois dont 1,000 au detriment des bureaux.

Il est évident que, malgré leur modestie, ces resultats sont encourageants. Mais ils demeurent insuffisants pour, d'une part, absorber les quelques 45,000 étudiants inscrits dans les écoles américaines d'architecture et, d'autre part, amplifier l'apport social de l'architecte. C'est pourquoi nous travaillons actuellement à une conceptualisation économique de la diversification de la profession susceptible de servir de base à des politiques universitaires appropriées. 


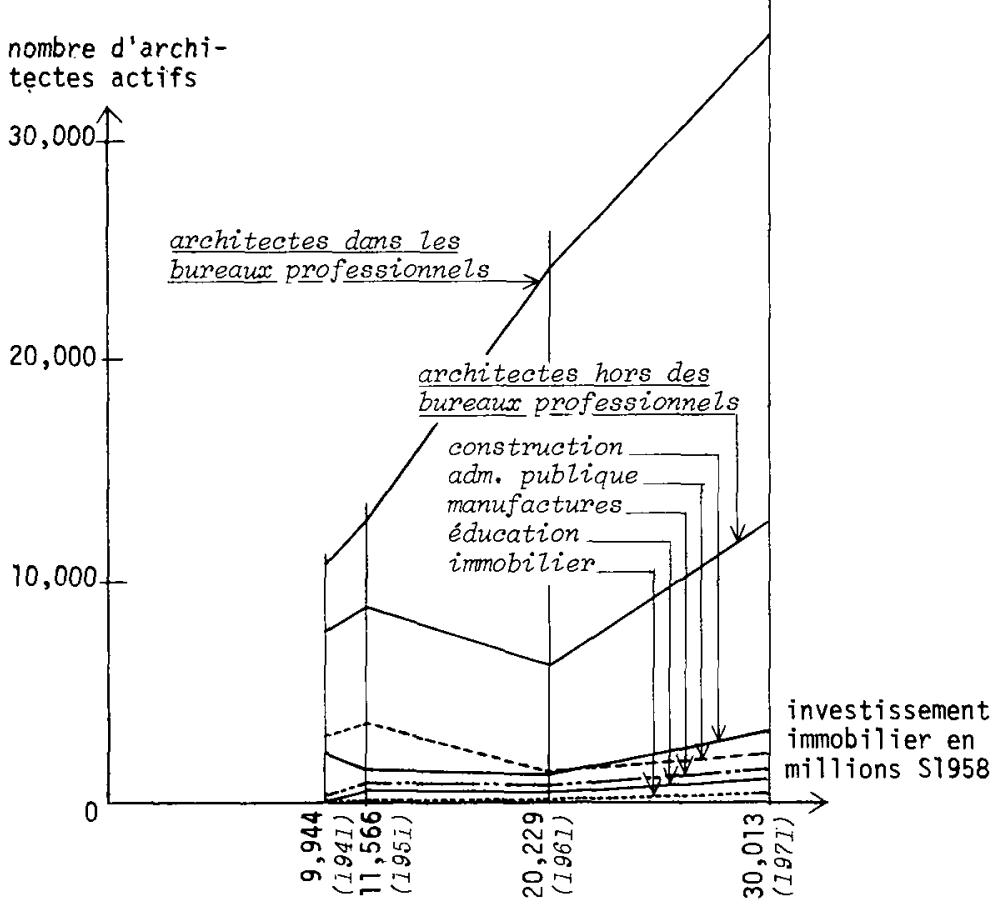

Graphique 8. Architectes actifs selon les industries et selon l'investissement immobilier aux années de recensement, U.S.A.

\section{Vers un schéma économique de la diversification des profils d'architecte}

Le cadre bâti est le résultat d'une activité qui implique la plupart des secteurs économiques. L'activité de ces secteurs peut être analysée sous l'angle de l'investissement, de la production et de l'usage ou consommation.

Les secteurs d'investissement sont traditionnellement ceux de l'activité économique auxquels on ajoute le logement ${ }^{(33)}$. Les investissements pour le cadre bâti couvrent les dépenses en construction. Cependant, les dépenses regroupées sous le titre de "real estate" comprennent non seulement le contrat de construction mais aussi l'acquisition des terrains, les conseils techniques, les frais de promotion et de mise en marché, etc. Les secteurs d'investissement figurent dans la deuxième colonne du tableau 9 , ci-après.

Quelle est la problématique de l'architecte vis-à-vis des secteurs d'investissement? Bien sûr, le fait qu'en général, la moitié des projets se fait sans appel à ses services. Mais aussi le fait que lorsqu'il intervient dans l'autre moitié, la décision d'investir a déjà été prise et la localisation, la programmation, voire les caractéristiques physiques générales du projet ont déjà été déterminées. En définitive, si l'architecte intervient, il le fait uniquement au niveau du design, quand ce n'est, comme on dit dans le jargon, au niveau de la décoration.

Pour ce qui est des secteurs de production, un simple coup d'oeil à la comptabilité inter-industrielle ${ }^{(34)}$ montre que la réalisation du cadre bâti est le résultat de l'activité 


\begin{tabular}{|c|c|c|c|}
\hline $\begin{array}{l}\text { secteurs d'activité } \\
\text { industrielle }\end{array}$ & $\begin{array}{c}\text { secteurs } \\
\text { d'immobilisation }\end{array}$ & $\begin{array}{l}\text { secteurs de produc- } \\
\text { tion du cadre bati }\end{array}$ & $\begin{array}{l}\text { secteurs d'usage \& } \\
\text { gestion cadre bati }\end{array}$ \\
\hline $\begin{array}{l}\text { 1. agriculture et } \\
\text { péche }\end{array}$ & $\begin{array}{l}\text { agriculture et } \\
\text { pêcche }\end{array}$ & - & \multirow{9}{*}{ Centreprises } \\
\hline 2. foresterie & foresterie & bois de sciage & \\
\hline $\begin{array}{l}\text { 3. mines, carriêres, } \\
\text { puits de pétrole }\end{array}$ & $\begin{array}{l}\text { mines, carriẽres, } \\
\text { puits de pétrole }\end{array}$ & $\begin{array}{l}\text { matiêres premières } \\
\text { (sauf bois) }\end{array}$ & \\
\hline 4. manufactures & manufactures & $\begin{array}{l}\text { tous matériaux de } \\
\text { construction sauf } \\
\text { bois de sciage et } \\
\text { béton fabriqué sur } \\
\text { place }\end{array}$ & \\
\hline 5. construction & $\begin{array}{l}\text { industrie de la } \\
\text { construction }\end{array}$ & $\begin{array}{l}\text { entreprise gënéra- } \\
\text { le et corps d'état }\end{array}$ & \\
\hline $\begin{array}{l}\text { 6. transports, com- } \\
\text { munications et } \\
\text { autres utilités }\end{array}$ & $\begin{array}{l}\text { services d'utili- } \\
\text { té publique }\end{array}$ & $\begin{array}{l}\text { transports, commu- } \\
\text { nications, énergie, } \\
\text { eau, etc... }\end{array}$ & \\
\hline 7. commerce & $\begin{array}{l}\text { commerce (sauf } \\
\text { services commerc.) }\end{array}$ & $\begin{array}{l}\text { commerce de maté- } \\
\text { riax de construc. }\end{array}$ & \\
\hline $\begin{array}{l}\text { 8. finance, assuran- } \\
\text { ces et immobilier }\end{array}$ & $\begin{array}{l}\text { finance, assuran- } \\
\text { ces et immobilier } \\
\text { (sauf logement) }\end{array}$ & $\begin{array}{l}\text { services financiers } \\
\text { et assurances a la } \\
\text { construction, pro- } \\
\text { motion immobilière }\end{array}$ & \\
\hline $\begin{array}{l}\text { 9. services aux en- } \\
\text { treprises, com- } \\
\text { merciaux et per- } \\
\text { sonnels }\end{array}$ & $\begin{array}{l}\text { services aux en- } \\
\text { treprises, commer- } \\
\text { ciaux et person- } \\
\text { nels }\end{array}$ & $\begin{array}{l}\text { services techniques } \\
\text { architectes, in- } \\
\text { gênieurs, géomé- } \\
\text { tres, etc... } \\
\text { services administr. } \\
\text { comptabilite, é- } \\
\text { valuation, esti- } \\
\text { mation, gestion, } \\
\text { etc... }\end{array}$ & \\
\hline $\begin{array}{l}\text { 10. administration } \\
\text { publique }\end{array}$ & $\begin{array}{l}\text { administration pu- } \\
\text { blique }\end{array}$ & $\begin{array}{l}\text { administration pu- } \\
\text { blique (contrôle) }\end{array}$ & $\begin{array}{l}\text { administration pu- } \\
\text { blique }\end{array}$ \\
\hline 11. divers & logement & travaux domestiques & ménages et public \\
\hline
\end{tabular}

Graphique 9. Secteurs d'investissement, de production et 'd'usage du câdre bâti.

d'un grand nombre de secteurs économiques. Ainsi, une fois que la décision d'investir a été prise, ces secteurs entrent directement ou indirectement en jeu aux fins de la production de l'édifice envisagé. En prenant comme repère le contrat de construction, on retrouve les secteurs situés "en amont" tels que la finance, les services techniques et l'administration publique (contrôle) et les secteurs "en aval" tels que l'industrie de la construction, les manufactures de matériaux ou la foresterie. Tous ces secteurs figurent à la troisième colonne du tableau 9 , ci-avant. 
A l'exception du secteur des services techniques où se trouvent précisément les bureaux d'architectes et, dans une certaine mesure, du secteur de l'administration publique, la présence d'architectes dans la production du cadre bâti est encore rare, malgré les progrès signalés plus haut.

Enfin, les secteurs d'activité mentionnés sont eux-mêmes les usagers du cadre bâti. Ceci pose toute la question de la gestion du patrimoine (utilisation optimale de l'espace, conservation, réanimation, etc.) au niveau des trois grands secteurs de consommation: les entreprises, l'administration publique et les ménages.

\section{CONCLUSION}

L'optique de la diversification que nous venons d'esquisser soustend une spécificité de l'architecte. Or, à défaut d'autre élément, cette spécificité finit toujours par se reporter à une fonctionnalité, ou plus concrètement, à une définition de tâches. Dans le cas de l'architecte, la définition retenue par les classifications officielles des professions est particulièrement traditionnelle (6). Elle correspond à un mode de pratique dont nous venons d'apprécier les limites. Elle correspond aussi à une problématique décalquée sur le "pattern" de la croissance économique.

Il est clair qu'à long terme, l'activité architecturale devra se définir non seulement à travers une diversité de secteurs d'action, comme le fait la diversification, mais encore à travers une diversité de fonctions. En d'autres termes, aux deux questions qui et où, il faudra ajouter la réponse à une troisième question: que fait l'architecte?

Cette dernière question est d'autant plus difficile que la société toute entière est à un tournant que beaucoup souhaitent décisif. Les sociétés industrialisées, aux prises avec des problèmes économiques inattendus et soumises à une mutation sociale rapide, se tournent de plus en plus vers de nouveaux modèles de comportement. Il n'est pas impossible que la société cherche sa désaliénation en se restructurant autour de concepts hier impensables, tels la "société de conservation"(35) ou l" "économie de l'information"(36). Il est clair, en tout cas, que l'évolution du cadre bâti sera tributaire du genre de société qui prévaudra, bien que la thèse de "la ville: produit de la société" soit, elle-même, réversible ${ }^{(37)}$.

L'ajustement des fonctions de l'architecte à l'évolution du cadre bâti ne peut être envisagé que dans un sens prospectif, donc à long terme. Cet ajustement sera par ailleurs la mesure de la contribution de l'architecte au changement social. Faut-il encore qu'il ait l'occasion de le faire. C'est précisément le rôle de la diversification que de la lui fournir. Le reste, c'est l'avenir.

\section{Notes Bibliographiques}

1. Annuaire statistique de l'UNESCO, 1972.

2. The Carnegie Commission on Higher Education, College Graduates and Jobs, McGraw-Hill, N.Y., 1973.

3. T.W. Schultz The Economics Value of Education, Columbia Univ. Press, N.Y., 1963.

4. Ceci explique, dans la prespective développée par E. Denison (note 21), le faible niveau comparé des indicateurs de développement de la production du cadre bâti. 
5. M. MacEwen, Crisis in Architecture in RIBA Journal, avril 1974.

6. Classification internationale standard des professions, B.I.T., Genève, 1969.

7. M. Vermot-Gauchy, Offre et demande d'ingénieurs, in Textes choisis en économie de l'éducation, UNESCO, Paris, 1968.

8. H. Correa, J. Tinbergen, Quantitative Adaptation of Education to Accelerated Growth, in Kyklos, vol. XV, 1962, p. 772.

9. R. Pressat, L'analyse démographique, P.U.F., Paris, 1973, Ch. 5.

10. Excepté au Royaume-Uni, où les architectes oeuvrant hors des bureaux représentent $50 \%$ du total. Cependant, cette proportion comprend les agences gouvernementales et celles des villes nouvelles.

11. R. Vergès-Escuin, Les architectes dans l'activité économique, Université de Montréal, 1976.

12. Le modèle a été construit à partir des données de 1953 à 1973, issues d'une part de l'enquête annuelle de la revue Progressive Architecture auprès des bureaux d'architecte américains et d'autre part, des montants des contrats de construction aux U.S.A., relevés annuellement par F.N. Dodge Division.

13 Notations générales classiques. Voir par exemple, R.J. Wonnacott, T.H. Wonnacott, Econometrics, John Wiley, N.Y., 1970.

14. Jeannine Perrault, Migration interprovinciale, Document de travail no. 8, Statistique Canada, 1972.

15. Ph. Langley Aspects de la profession architecturale, in Architecture d'aujourd'hui, no. 170, 1973.

En général, il y aujourd'hui dans les différents pays industrialisés autant d'étudiants en architecture que d'architectes dans la pratique (U.S.A., France, Canada, Japon. . .). Certains pays en ont même trois fois plus comme l'Italie: Rome possède plus de 12,000 élèves-architectes. Au Mexique, la seule ville de Mexico en compte 7,000, et. . .

16. Les cycles visibles sur le graphique correspondent aux cycles dits "longs" ( $8-9$ ans), lesquels sont à distinguer des cycles "Kuznets" (18-20 ans) et des cycles "courts" (2-4 ans). Voir par exemple, M. Abramovitz, Evidence of Long Swings in Aggregate Construction Since Civil War, N.B.E.R., N.Y., 1964.

17. W. Tochtermann, M. Weill, 1976, Année de l'Habitat, Université radiophonique et télévisuelle internationale, mai 1976.

18. Si l'on s'en tient uniquement à la littérature américaine, on peut distinguer trois grands courants. Tout d'abord, celui des cycles de longue durée: Riggleman (1933), Newmann (1935), Long (1940), Colean \& Newcomb (1952) Blank (1954), Kuznets (1961), Abramovitz (1964). . Voir à ce propos Historical Statistics of the United States, Colonial Times to 1970, Wash. 1975 ou R.E. Lipsey \& D. Preston, Source Book of Statistics Relating to Construction, N.B.E.R., N.Y., 1966. L'analyse spectrale a repris récemment ce thème grâce à Aldeman, Howrey et surtout Cargill, Construction Activity and Secular Change in the United States in Applied Economics, 1971, pp. 85-97.

Un deuxième courant est centré sur le thème de l'élasticité de la demande d'habitation et les cycles de courte durée. Voir les auteurs de Study of Mortgage Credit (1958) ainsi que Grebbler (1959), Guttentag (1961), L.B. Smith (1972) et autres. Une synthèse de ce courant figure dans A. D'Amours et L. Nadeau, L'économie de l'habitation: une revue de la littérature in L'actualité économique, avril-juin 1974.

Enfin, le troisième s'est occupé du stock de capital. Grebbler, Blank \& Winning (1956), Firestone (1951, Canada), Hood \& Scott (1957, Canada), Kuznets (1961), etc. Plus tard, ces études ont été perfectionnées: J.C. Musgrave, Fixed Non-residential Business and Residential Capital in the United States, 1925-1975, B.E.A., Wash. 1975. P. Koumanakos, Alternative Estimates of Non-residential Capital in Canada, Statistics Canada, Ottawa, 1975.

19. Dans certaines situations de marginalité, cette condition ne se remplit parfois qu'avec des décalages temporels très importants.

20. Etude réalisée pour l'Union internationale d'architectes en vue de son XIIIe Congrès mondial à Mexico en 1978.

21. La méthode utilisée est celle des stocks bruts par l'inventaire perpétuel, R.W. Goldsmith, A Perpetual Inventory of National Wealth, N.B.E.R., N.Y., 1951. 
22. Par exemple, le Québec, qui est passé en 50 ans de 2.6 à 6.2 millions d'habitants, semble avoir atteint son niveau de stagnation. Montréal, que l'on annonçait comme une métropole de 7 millions d'habitants en l'an 2,000, n'en aura guère plus de 3, si elle les atteint un jour!

23. En fait, le retrait du stock ne se fait pas ponctuellement mais selon une courbe normale "Winfrey S3". Cf. Koumanakos, note (18).

24 A. Page, L'Economie de l'éducation, P.U.F., Paris, 1971, p. 10-15.

25. M. Blaug An Introduction to Economics Education, Pergamon Press, Londres, 1970.

26. E.F. Denison, The Sources of Economic Growth in the U.S. and the Alternative before us, Committee for Economic Development, Wash., 1962.

27. H.R. Bowen, The Manpower vs the Free-Choice Principle in University Affairs, jan. 1974.

28. Un bon exemple, malgré son ésotérisme, est l'article Architects Aspects in Architectural Design, juil. 1976, p. 268-295.

29. D. Hunt Jr., Comprehensive Architectural Practice, in Journal of American Institute of Architects, juin 1962, p. 77-86.

30. U.S. Population Census, 1940: The Labor Force, Occupation Characteristics, (sample statistics), tab 3. 1950: vol IV, part I, ch C, tab 2. 1960: PC (2) FC, tab 2. 1970: PC (2) FC, tab 8.

31. U.S. Department of Commerce, Value of New Construction Put in Place, Washington, mensuel.

32. R. Vergès-Escuin, Historical Evoluation of U.S. Building Capital Stock vs Income, 1875-1975, Montréal, 1977.

33. Voir par exemple, Department of Commerce, The National Income and Product Accounts of the U.S. (selon l'activité industrielle)

34. Aux U.S.A., Department of Commerce, Input/Output Structure of the U.S. Economy, 1963, in Survey of Current Business, nov. 1969.

35. Voir par exemple, K. Valaskakis, P.S. Sindell, J.G. Smith, La société de conservation sélective, vol. I, Groupe Gamma, Montréal, 1976.

36. J. Attali, La parole et l'outil, P.U.F., Paris, 1975.

37. M. Castells, Y a-t-il une sociologie urbaine? in Sociologie du travail, no. 1, 1968. 